\title{
Effects of Concentrated Growth Factor and Hyaluronic Acid in an Experimental Model of Acute Traumatic Tympanic Membrane Perforation
}

\author{
(D) Hüseyin Sarı1, (D) Yavuz Atar1, (D) Selma Şengiz Erhan², (D) Ruslan Hummatov1, (D) Öykü Izel Başaran¹, (D) Semih Karaketir3, \\ (i) Deniz Hancı1, (D) Güler Berkiten ${ }^{1}$, (D) Yavuz Uyar \\ 1 University of Health Sciences Turkey, Prof. Dr. Cemil Taşcıoğlu City Hospital, Clinic of Otorhinolaryngology, Istanbul, Turkey \\ 2University of Health Sciences Turkey, Prof. Dr. Cemil Taşcıoğlu City Hospital, Clinic of Pathology, Istanbul, Turkey \\ ${ }^{3}$ Bulanık State Hospital, Clinic of Otorhinolaryngology, Muş, Turkey
}

\section{Abstract}

Objective: The aim of this study is to investigate the efficacy of concentrated growth factor (CGF) and hyaluronic acid (HA) application on the healing of acute tympanic membrane (TM) perforation.

Methods: A total of 30 male albino rats were included in the study. The animals were randomly divided into three groups with 9 rats in each group (A, B, C). A CGF was applied to the perforated TMs of rats in group A. A gelfoam particle soaked in 1\% hyaluronan was applied to the perforations in group B. The rats in group C were left to heal spontaneously. All rats were kept under control for 21 days. TM healing was evaluated. On the $21^{\text {st }}$ day, all rats were decapitated for histopathological evaluation.

Results: In group A, 18 of the 18 TM perforations were closed and the mean healing time was 12.11 days. In group B, 18 of the 18 TM perforations were closed and the mean healing time was 15.05 days. In group C, 16 of 18 TM perforations were closed and the mean closure time was 17.11 days. There was a statistically significant difference in the mean TM closure time in all groups at the end of the $21^{\text {st }}$ day $(p=0.001)$.

Conclusion: In this study, we used autologous CGF and HA in the treatment of acute TM perforations in rats. We observed that the application of both products was a more effective method than the control group. We think that using these methods, which are easy, inexpensive, and have low patient morbidity, as graft or graft support in chronic TM perforations will be beneficial and successful results will increase.

Keywords: Concentrated growth factor, hyaluronic acid, tympanic membrane

\section{INTRODUCTION}

Tympanic membrane (TM) perforation often has different causes, such as trauma and infection. If the perforation is not healed, important problems such as chronic otitis media and hearing loss may occur. The reported incidence of TM perforation ranged from $1 \%$ to $4 \%$ (1). Perforations often heal spontaneously unless chronic inflammation develops; however, with chronic perforation, surgical repair is usually required (2).
In traumatic perforations, especially when less than a quarter of the membrane is perforated, approximately $90 \%$ of them heal spontaneously, while grafting is often required to repair large perforations (3).

Healing of perforated TM after acute trauma includes epithelial proliferation and migration, fibroblast proliferation, angiogenesis, and tissue remodeling. The epithelial layer first forms a bridge over the wound, the surface of the fibrous layer 
is closed, and the TM takes its final form (4). Different cytokines and growth factors are released from the wound site during the acute stage of wound healing. Many otolaryngologists perform microsurgical procedures to accelerate the healing process. These include early patching, surgery, and use of biomaterials such as hyaluronate, epidermal growth factor (EGF), fibroblast growth factor, platelet-derived growth factor, and transforming growth factor $(5,6)$. Autogenous materials may be used more frequently in TM repairs because of their positive effects on wound healing quantity and quality. Various animal experimental studies have examined the effects of these growth factors on wound healing. These studies have helped us learn the healing process of TM perforations in humans.

This study aimed to investigate the effectiveness of concentrated growth factor (CGF) and hyaluronic acid (HA), which can be obtained by a simple and inexpensive method, on the healing of acute TM perforation.

\section{METHODS}

This study was approved by the Animal Research Ethics Committee of Bezmialem Vakif University Medical Faculty (approval date: 21.03.2019, approval no. 2019/91). Thirty male albino rats with bilateral normal TM (weight, 250-300 g) breeding in the multidisciplinary research laboratories were examined and included in the study.

\section{Concentrated Growth Factor Preparation}

Of the 30 animals, three were used for CGF preparation. Then, 4 $\mathrm{mL}$ of intracardiac blood were collected from the three rats and placed in test tubes without anticoagulants. These tubes were centrifuged (MEDIFUGE TM, Silfradent Srl, S. Sofia, Italy) at 2700 rpm for $2 \mathrm{~min}, 2400 \mathrm{rpm}$ for $4 \mathrm{~min}, 2700 \mathrm{rpm}$ for $4 \mathrm{~min}$, and 3 $\mathrm{min}$ at $3000 \mathrm{rpm}$. The aim was to obtain a dense fibrin matrix containing growth factors at a higher rate by centrifuging the samples at different speeds and times. After the procedure, four layers were obtained from bottom to top: Red blood cell layer, growth factor and stem cell layer (CGF), buffy coat layer, and serum layer. Then, the CGF gel layer was separated using sterile surgical scissors and placed over the target site (7).

\section{Surgical Procedure}

The animals were randomly divided into three groups with nine rats in each group (groups $\mathrm{A}-\mathrm{C}$ ). Ketamine hydrochloride (40 mg/kg) and xylazine hydrochloride $(5 \mathrm{mg} / \mathrm{kg}$ ) were administered intraperitoneally to anesthetize all animals. In the otomicroscopic examination, a perforation was created using a pike in the posterosuperior quadrant of both TMs in all rats.
CGF was applied to the perforated ears in group A. A 1.5-2 mmdiameter gelfoam particle soaked in $10 \mu \mathrm{L}$ of $1 \%$ hyaluronan (Healon, Pharmacia AB, Sweden) was applied to the perforations in group B. No intervention was made to the perforated ears in group C, and they were left to heal spontaneously. All rats were observed in their groups in cages with access to water and food. The animals were examined microscopically daily under inhaled isoflurane anesthesia until the animals were sacrificed or 21 days had passed to define the time of perforation closure.

\section{Histopathological Examination}

On day 21, after intraperitoneal pentobarbital injections (80 mg/ $\mathrm{kg}$ ), all rats were decapitated. Their external ears were separated from the osteocartilaginous connections, and each bulla was opened. TMs were removed together with the bone annulus. All ear samples were stored in $10 \%$ formaldehyde solutions and sent to the pathology laboratory for histological evaluation. In total, 52 surgical specimens fixed in formaldehyde were decalcified in formic acid. Sections were embedded in paraffin. Tissue blocks were cut into 5-mm-thick slides, treated with hematoxylin and eosin, and then examined under a light microscope. Fibrosis, neovascularization, inflammation, and edema in tissue samples were evaluated in histopathological examination. All examinations were performed by the same pathologist without knowing which tissue sample belonged to which group. Changes in parameters were scored as $(-),(+),(++)$, or $(+++)$. Negative showed no change; $(+),(++)$, and $(+++)$ scores showed mild, moderate, and significant changes, respectively. No rats died during the study period.

\section{Statistical Analysis}

In the evaluation of the study findings, IBM SPSS Statistics 22 (IBM Corp., Armonk, NY, USA) program was used for statistical analysis. While evaluating the study data, the conformity of the parameters to the normal distribution was evaluated with the Shapiro-Wilk test. In addition to statistical methods (mean, standard deviation, and frequency), the Kruskal-Wallis test was used for the comparison of the parameters that did not show a normal distribution in the comparison of quantitative data, between more than two groups. The chi-square test was used to compare qualitative data. Significance was evaluated at the level of $p<0.05$.

\section{RESULTS}

The closure process of TMs was examined microscopically for 21 days in a total of 27 rats. In group A, 18 of the 18 TM perforations in which CGF was applied were closed, and the mean healing 
time was 12.11 days. In group B, 18 of the 18 TM perforations in which HA was applied were closed, and the mean healing time was 15.05 days. In group C, whose perforations were left to spontaneous heal, 16 of $18 \mathrm{TM}$ perforations were closed, and the mean closure time was 17.11 days (Table 1). One of the rats in the control group, which was left to heal spontaneously, did not heal in both TMs.

The mean closure times were evaluated in all groups after 21 days, and a significant difference was observed ( $p=0.001$ ) (Table 1). When the difference in the mean closure time between the groups was compared, it was shorter in group A than in groups $B$ and $C$, and the differences were found significant ( $p=0.004, p=0.001$, respectively). It was also slightly shorter in group B than in group $C(p=0.037)$ (Table 1). Histopathological evaluation initiated after 21 days of TM recovery evaluation revealed no significant difference between all animal groups in terms of edema, neovascularization, fibrosis, and inflammation parameters (Table 2).

\section{DISCUSSION}

TM perforations in humans appear in various sizes depending on the causes, such as infections, accidents, explosion, slap injury, or instrument-induced injury. Even a very small perforation can negatively affect people's daily quality of life. Approximately $90 \%$ of TM perforations heal spontaneously within 7-21 days, depending on their size (4). Following an acute trauma to the TM, an exudative reaction begins first at the edges of the perforation. After a while, a keratin migration starts from the external squamous epithelial layer of the TM to the center of the perforation. Crust layer formation protects the underlying tissue, providing a suitable basis for cell migration and the healing process. Closure of the TM defect initially occurs through the keratin layer, then squamous epithelial cells fuse, and a supporting connective tissue closes the perforation (8).

At present, myringoplasty is an effective procedure commonly used to repair TM perforations. Many graft types are used in myringoplasty. These grafts should be biocompatible and easy to obtain. In perforation repair, while autografts (temporal muscle fascia, tragal cartilage, and fat tissue), gel films, and paper patches are used for stromal support, silver nitrate cauterization and trichloride acetic acid can be applied to the perforation borders. The TM structures of humans and rats have similar histological features (9). Considering that similar results can be obtained in humans, rats are generally used in experimental studies on TM. Animals studies have shown the presence of stem cells in the annulus and manubrium malleus of TM. These cells are involved in the repair of TM owing to their high proliferation properties (4). Platelet-rich products are used in many areas in graft and wound treatment, as they promote accelerated angiogenesis, chemotaxis, mitosis, and proliferation of stem cells (10). To benefit from the effects of these products in wound healing, many types of platelet concentrate products have been used for years. These products take various names depending on their contents, centrifugation methods, and presence or absence of an anticoagulant in the production techniques. Platelet-rich plasma (PRP), platelet-rich fibrin (PRF), and CGF are some of these products. Studies on topical HA, EGF, PRP, PRF, and many other product applications have been reported in the treatment of traumatic TM perforations (11). Yeo et al. (12) reported that mouse TM perforations showed better closure than the control group with the application of a 2-ug platelet-derived growth factor. Mondain et al. (13) showed that the application of fibroblast growth factor to mouse TM perforation resulted in faster closure. By contrast, O'Daniel et al. (14) studied pigs and showed that EGF receptors are present in all three TM layers, especially the squamous epithelial layer.

PRP and PRF were first defined by Dohan et al. (15) by dividing them into two different groups according to their production methods and contents. El-Anwar et al. (16) performed tympanoplasty with conchal cartilage for dry and large perforated TM 64 patients. In half of the cases, they applied PRP during the procedure. They observed a higher graft retention rate and a lower infection rate in the PRP group. Another similar study showed better graft success rates using PRP-impregnated gel foam in tympanoplasty (17). Erkilet et al. (18) applied PRP to perforated TMs in rats and reported that PRP had positive effects on healing and shortened the recovery time. The main

Table 1. Closure time of the study and control groups

\begin{tabular}{|c|c|c|c|c|c|c|c|}
\hline & \multicolumn{5}{|c|}{ Closure time (day) } & \multirow[b]{2}{*}{$\mathbf{p}$} & \multirow[b]{2}{*}{ Pairwise comparisons of the groups } \\
\hline & n (ear) & Minimum & Maximum & Mean & SD & & \\
\hline Group A (CGF) & 18 & 9 & 15 & 12.11 & 1.94 & \multirow{2}{*}{0.001} & \multirow{2}{*}{$\begin{array}{l}\text { Group A-B; } p=0.004 \\
\text { Group B-C; } p=0.037 \\
\text { Group A-C; } p=0.001\end{array}$} \\
\hline Group C (control) & 16 & 12 & 20 & 17.11 & 1.68 & & \\
\hline
\end{tabular}

Kruskal-Wallis test $\mathrm{p}<0.05$, Pairwise comparison: Mann-Whitney with Bonferroni correction, SD: Standard deviation, CGF: Concentrated growth factor, HA: Hyaluronic acid 
generations of autologous platelet concentrate produced by centrifugation of venous blood at different rates are PRP, PRF, and CGF. CGF, which is the newest autologous platelet concentrate, was first described by Sacco (19). The rationale for this product is related to the in situ administration of multiple autogenous growth factors, as well as specific fibrin scaffold formation. Lei et al. (20) conducted a comparative study between advanced PRF (A-PRF), a variant of PRF, and CGF. They showed that A-PRF had a looser fibrin network than CGF, and stimulation of a sustained release of growth factor for 10 days was comparable in both products. Another comparative study showed that platelet and growth factor concentrations in A-PRF were comparable to CGF, but A-PRF and CGF had higher platelet and growth factors than PRP and plasma-rich growth factors (21). Contents of PRF and CGF are similar, but CGF is rich in growth factors and has a denser fibrin matrix. These materials have properties that increase soft tissue and bone healing (22). Membranes obtained from these biomaterials can be used as graft material in otological applications such as TM repair. CGF or PRF is preferred over PRP because no anticoagulants are added, and their membranes provide better biomechanical resistance. This event is valuable in terms of graft survival.
Ensari et al. (23) used PRF as graft material in rats and reported that the mean recovery time of TM perforation was $10.3 \pm 2.18$ days in the study group and $17 \pm 2.40$ days in the control group $(p<0.05)$. Gür et al. (24) applied PRF in 30 of 60 patients with acute TM trauma and applied paper patches to the other 30 patients. They observed perforation closure in $93 \%$ of the patients in the PRF group and 83\% of the patients in the paper group. They did not observe a significant difference in the healing rates of TMs. They reported 7.8 as the mean closure time of TMs in the PRF group. In addition, Habesoglu et al. (25) reported that the use of PRF in acute traumatic TM perforations resulted in significantly better recovery rates and times. In our study, we examined the healing process of TM perforations we have created in 18 rats for 21 days to observe the effect of CGF on traumatic TM perforation. Subsequently, 18 of the 18 TM perforations in which we applied CGF were closed, and the mean recovery time was 12.11 days. In the control group, whose perforations were left to heal spontaneously, 16 of 18 TM perforations were closed, and the mean closure time was 17.11 days (Table 1). A significant difference was found between the two groups in terms of mean recovery times of TM $(p=0.001)$.

\begin{tabular}{|c|c|c|c|c|c|c|c|}
\hline & \multicolumn{2}{|c|}{ Group A (CGF) } & \multicolumn{2}{|c|}{ Group B (HA) } & \multicolumn{2}{|c|}{ Group C (control) } & \multirow[b]{2}{*}{$p$} \\
\hline & $n$ & $\%$ & $n$ & $\%$ & $n$ & $\%$ & \\
\hline \multicolumn{8}{|c|}{ Edema } \\
\hline - & 11 & 61.1 & 15 & 83.3 & 15 & \begin{tabular}{|l|}
83.3 \\
\end{tabular} & \multirow{4}{*}{0.052} \\
\hline+ & 6 & 33.3 & - & - & 2 & $\mid 11.1$ & \\
\hline++ & 1 & 5.6 & 3 & 16.7 & 1 & 5.6 & \\
\hline+++ & - & - & - & - & - & - & \\
\hline \multicolumn{8}{|c|}{ Neovascularization } \\
\hline - & 16 & 88.9 & 17 & 94.4 & 18 & 100 & \multirow{4}{*}{0.347} \\
\hline+ & 2 & 11.1 & 1 & 5.6 & - & & \\
\hline++ & - & - & - & - & - & & \\
\hline+++ & - & - & - & - & - & & \\
\hline \multicolumn{8}{|c|}{ Fibrosis } \\
\hline - & - & - & 5 & 27.8 & 6 & 33.3 & \multirow{4}{*}{0.113} \\
\hline+ & 11 & 66.7 & 10 & 55.6 & 9 & 50 & \\
\hline++ & 4 & 22.2 & 3 & 16.7 & 3 & 16.7 & \\
\hline+++ & 3 & 11.1 & - & - & - & - & \\
\hline \multicolumn{8}{|c|}{ Inflammation } \\
\hline- & 18 & 100 & 18 & 100 & 17 & \begin{tabular}{|l|}
94.4 \\
\end{tabular} & \multirow{4}{*}{0.361} \\
\hline+ & - & - & - & - & 1 & 5.6 & \\
\hline++ & - & - & - & - & - & - & \\
\hline+++ & - & - & - & - & - & - & \\
\hline
\end{tabular}


CGF, like other platelet concentrate products, accelerates wound healing, because it contains growth factors and cytokines. Since it is an autologous product, it does not pose a risk of infection and immune response. It also slowly releases growth factors into the environment through the fibrin network. It is easy and inexpensive to obtain (26). Recent studies with CGF are mostly on dental operations and bone tissue, and there are very few studies on TM. Sohn et al. (26) used CGF for maxillary sinus augmentation as well as implant surgery. They showed that CGF has an accelerating effect on new bone formation and soft tissue healing. In another case report, sinus perforation was treated with CGF, and new bone formation was demonstrated in the control evaluation performed with radiological examinations 6 months later (27). Talaat et al. (28) applied PRP, PRF, and CGF to the defects in 20 patients with bone defects caused by mandibular lesions. As a result, they reported that CGF is an economical and safe product that accelerates new bone formation. Topkara et al. (29) performed an animal study and showed that the viability of chopped cartilage covered with fascia, which is one of the important camouflage materials in rhinoplasty surgery, will increase when used with CGF. Zhao et al. (30) performed repeated local injections of CGF in patients with septal mucosal defects after rhinoplasty, and they observed complete closure in all patients, indicating that this technique is an easy and convenient procedure. Many surgeons have used adipose tissue as a graft, especially in the repair of small TM perforations. Fat graft myringoplasty is also a cost-effective, simple, and non-invasive surgery for TM perforations. However, fat grafting is a surgical procedure, albeit a minor one. CGF membrane application does not require any surgical procedure, and a blood sample from the patient is sufficient. In addition, CGF has a protective effect against infections.

Since fibrin obtained from platelet-rich materials contains proinflammatory cytokines, an increase in inflammation and fibrosis rates is expected in the areas where it is applied (31). Hu et al. (32) added PRP, PRF, and CGF to adipose tissues taken from rats and examined histologically. They observed richer vascularity and less fibrosis in the CGF added group compared with the PRP and PRF groups. Erkilet et al. (18) evaluated the improvement of TM perforations in rats in which PRP was applied, although it was not significant, fibroblastic reaction and neovascularization were higher in the study group on day 7 . Ensari et al. (23) evaluated the perforated TMs of the rats to which they applied PRF, and they reported that neovascularization was more frequent days 3,5 , and 7 , although it was not significant in the study group $(p>0.05)$. Herein, we performed the histopathological evaluation in the last week of our study. When the tissues were taken for histopathological examination on day 21 , no significant difference was found between all animal groups in terms of edema, neovascularization, fibrosis, and inflammation parameters (Table 2). In other studies, significant differences were found compared with the control groups in terms of these parameters; no difference was found between the groups because our histopathological evaluation was performed at a later stage.

$\mathrm{HA}$, which is routinely used in ophthalmologic surgery because of its viscoelastic properties, is involved in various processes of early wound healing such as cell migration, organization of granulation tissue in cell proliferation, moderation of the inflammatory response, and angiogenesis $(33,34)$. Local application of HA has positive effects in closing TM perforations in tympanoplasty and in reducing the length of hospital stay and cost (35). These effects regulate the healing of the fibrous layer by preventing dehydration of the perforation margins, providing a supportive environment for the keratin and hyperplastic epithelium that occurs during the healing process, and regulating functions such as mobility and phagocytic properties of polymorphonuclear leukocytes (36). Ozturk et al. (37) performed an experimental animal study with 24 male rats; they divided the rats into three groups as control rats, MeroGel rats, and daily topical HA rats. Perforations were closed in $70.8 \%, 91.7 \%$, and $100 \%$ in the control, MeroGel rats, and daily HA rats, respectively. They reported that the main role of HA in repairing the TM is to provide a moist, wound-healing environment to aid in the healing process. Kaur et al. (38) used topical application of $1 \%$ sodium hyaluronate to repair human chronic TM perforations and reported that $86.7 \%$ (26/30) of the patients responded positively to the treatment. Rivas Lacarte et al. (39) reported that with the topical application of $1 \%$ sodium hyaluronate in 16 patients with chronic TM perforations, the perforation size reduced in 12 (75.0 \%) patients; 6 (37.5\%) of whom showed complete healing, and 4 (25.0\%) showed no healing. Güneri et al. (6) evaluated the improvement of TM perforations with $\mathrm{HA}$ in another experimental animal study. While the mean closure time of TM perforation was $8.6 \pm 2$ days in the HA-treated group, it was $15 \pm 2$ days in the control group $(p=0.0432)$. Similarly, in our study, all TM perforations were closed in HA rats, but the mean closure time was 15.5 days. In our control group, this period was 17.11 days $(p=0.037)$. We think that these differences in mean recovery times are attributed to the number of rats used, localization of the perforations on the TM, and amount of HA applied. These findings obtained from rat studies suggest that HA application will have positive effects on the healing of acute traumatic TM perforations. In 
addition, a study on wound healing has shown that a moist wound environment accelerates the healing of both acute and chronic wounds and promotes the growth of new tissue (40). Therefore, generally speaking, topical application of HA in the repair of traumatic TM perforation may exhibit a better healing state, thanks to the moist environment it provides.

\section{CONCLUSION}

Few studies have focused on the use of PRF or CGF in the repair of TM perforations. In our study, we used autologous CGF membrane and $\mathrm{HA}$ in the treatment of acute perforations in rats. The application of both products was a more effective method than the control group. With these methods, which are easy, inexpensive, and have low patient morbidity, as graft or graft support in chronic TM perforations will be beneficial and promote successful results. The development of biological materials can assist or, in appropriate cases, replace conventional myringoplasty in repairing chronic TM perforations.

\section{Ethics}

Ethics Committee Approval: This study was approved by the Animal Research Ethics Committee of Bezmialem Vakif University Medical Faculty (approval date: 21.03.2019, approval no. 2019/91).

Informed Consent: Animal experiments.

Peer-review: Externally peer-reviewed.

\section{Authorship Contributions}

Concept: H.S., Y.A., Y.U., Design: H.S., Y.U., Data Collection or Processing: Ö.I.B., S.K., Analysis or Interpretation: Y.A., S.S.E., D.H., G.B., Literature Search: S.K., D.H., Y.U., Writing: H.S., Y.A.

Conflict of Interest: No conflict of interest was declared by the authors.

Financial Disclosure: The authors declared that this study received no financial support.

\section{REFERENCES}

1. Howard ML, Meyer AD, Bauer CA, Talavera F, Gianoli GJ, Slack CL. Middle ear tympanic membrane perforations. Medscape; 2012. http:// emedicine.medscape.com/article/858684-overview

2. Villar-Fernandez MA, Lopez-Escamez JA. Outlook for tissue engineering of the tympanic membrane. Audiol Res 2015;5:117.

3. Davidson BJ, Morris MS. The perforated tympanic membrane. Am Fam Physician 1992;45:1777-82.

4. Wang WQ, Wang ZM, Chi FL. Spontaneous healing of various tympanic membrane perforations in the rat. Acta Otolaryngol 2004;124:1141-4.
5. Lou Z, Wang Y. Evaluation of the optimum time for direct application of fibroblast growth factor to human traumatic tympanic membrane perforations. Growth Factors 2015;33:65-70.

6. Güneri EA, Tekin S, Yilmaz O, Ozkara E, Erdağ TK, Ikiz AO, et al. The effects of hyaluronic acid, epidermal growth factor, and mitomycin in an experimental model of acute traumatic tympanic membrane perforation. Otol Neurotol 2003;24:371-6.

7. Bozkurt Doğan \$, Öngöz Dede F, Ballı U, Atalay EN, Durmușlar MC. Concentrated growth factor in the treatment of adjacent multiple gingival recessions: a split-mouth randomized clinical trial. J Clin Periodontol 2015;42:868-75.

8. Glasscock III ME, Shambaugh Jr. GE. Closure of tympanic membrane perforations. In: Surgery of the Ear. 4th ed. Philadelphia: WB Saunders; 1990. p. 335-49.

9. Schmidt SH, Hellström S. Tympanic-membrane structure--new views. A comparative study. ORL J Otorhinolaryngol Relat Spec 1991;53:32-6.

10. Cortese A, Pantaleo G, Borri A, Caggiano M, Amato M. Platelet-rich fibrin (PRF) in implant dentistry in combination with new bone regenerative technique in elderly patients. Int J Surg Case Rep 2016;28:52-6.

11. Somers T, Goovaerts G, Schelfhout L, Peeters S, Govaerts PJ, Offeciers E. Growth factors in tympanic membrane perforations. Am J Otol 1998;19:428-34.

12. Yeo SW, Kim SW, Suh BD, Cho SH. Effects of platelet-derived growth factor-AA on the healing process of tympanic membrane perforation. Am J Otolaryngol 2000;21:153-60.

13. Mondain M, Saffiedine S, Uziel A. Fibroblast growth factor improves the healing of experimental tympanic membrane perforations. Acta Otolaryngol 1991;111:337-41.

14. O'Daniel TG, Petitjean M, Jones SC, Zogg J, Martinez SA, Nolph MB, et al. Epidermal growth factor binding and action on tympanic membranes. Ann Otol Rhinol Laryngol 1990;99:80-4.

15. Dohan DM, Choukroun J, Diss A, Dohan SL, Dohan AJ, Mouhyi J, et al. Platelet-rich fibrin (PRF): a second-generation platelet concentrate. Part I: technological concepts and evolution. Oral Surg Oral Med Oral Pathol Oral Radiol Endod 2006;101:e37-44.

16. El-Anwar MW, El-AhI MA, Zidan AA, Yacoup MA. Topical use of autologous platelet rich plasma in myringoplasty. Auris Nasus Larynx 2015;42:365-8.

17. Saeedi M, Ajalloueian M, Zare E, Taheri A, Yousefi J, Mirlohi SMJ, et al. The Effect of PRP-enriched gelfoam on chronic tympanic membrane perforation: a double-blind randomized clinical trial. Int Tinnitus J 2017;21:108-11.

18. Erkilet E, Koyuncu M, Atmaca S, Yarim M. Platelet-rich plasma improves healing of tympanic membrane perforations: experimental study. J Laryngol Otol 2009;123:482-7.

19. Sacco L. Lecture. In International Academy of Implant Prosthesis and Osteoconnection; Lecture: Sersale, Italy, 2006.

20. Lei L, Yu Y, Han J, Shi D, Sun W, Zhang D, et al. Quantification of growth factors in advanced platelet-rich fibrin and concentrated growth factors and their clinical efficacy as adjunctive to the GTR procedure in periodontal intrabony defects. J Periodontol 2020;91:462-72.

21. Masuki H, Okudera T, Watanebe T, Suzuki M, Nishiyama K, Okudera $H$, et al. Growth factor and pro-inflammatory cytokine contents in plateletrich plasma (PRP), plasma rich in growth factors (PRGF), advanced platelet-rich fibrin (A-PRF), and concentrated growth factors (CGF). Int J Implant Dent 2016;2:19.

22. Rodella LF, Favero G, Boninsegna R, Buffoli B, Labanca M, Scarì G, et al. Growth factors, CD34 positive cells, and fibrin network analysis in concentrated growth factors fraction. Microsc Res Tech 2011;74:772-7. 
23. Ensari N, Gür ÖE, Öztürk MT, Süren D, Selçuk ÖT, Osma Ü. The effect of platelet-rich fibrin membrane on the repair of perforated tympanic membrane: an experimental study. Acta Otolaryngol 2017;137:695-9.

24. Gür ÖE, Ensari N, Öztürk MT, Boztepe OF, Gün T, Selçuk ÖT, et al. Use of a platelet-rich fibrin membrane to repair traumatic tympanic membrane perforations: a comparative study. Acta Otolaryngol 2016;136:1017-23.

25. Habesoglu M, Oysu C, Sahin S, Sahin-Yilmaz A, Korkmaz D, Tosun A, et al. Platelet-rich fibrin plays a role on healing of acute-traumatic ear drum perforation. J Craniofac Surg 2014;25:2056-8.

26. Sohn DS, Moon JW, Moon YS, Park JS, Jung HS. The use of concentrated growth factors (CGF) for sinus augmentation. Journal Oral Implant 2009;38:25-38.

27. Sohn DS, Jung HS, Kim KH, Song KJ, An HW, Min KH. Removal of displaced foreign body from the maxillary sinus using replaceable bony windows and saline irrigation, followed by suctioning of the foreign body. Implant Dent 2011;20:112-7.

28. Talaat WM, Ghoneim MM, Salah O, Adly OA. Autologous bone marrow concentrates and concentrated growth factors accelerate bone regeneration after enucleation of mandibular pathologic lesions. J Craniofac Surg 2018;29:992-7.

29. Topkara A, Özkan A, Özcan RH, Öksüz M, Akbulut M. Effect of concentrated growth factor on survival of diced cartilage graft. Aesthet Surg J 2016;36:1176-87.

30. Zhao QM, Gao J, Huang XX, Chen XP, Wang X. Concentrated growth factors extracted from blood plasma used to repair nasal septal mucosal defect after rhinoplasty. Aesthetic Plast Surg 2020;44:511-6.

31. Dohan DM, Choukroun J, Diss A, Dohan SL, Dohan AJ, Mouhyi J, et al Platelet-rich fibrin (PRF): a second-generation platelet concentrate. Part
III: leucocyte activation: a new feature for platelet concentrates? Oral Surg Oral Med Oral Pathol Oral Radiol Endod 2006;101:e51-5.

32. Hu Y, Jiang $\mathrm{Y}$, Wang M, Tian W, Wang $\mathrm{H}$. Concentrated growth factor enhanced fat graft survival: a comparative study. Dermatol Surg 2018;44:976-84.

33. Maár N, Graebe A, Schild G, Stur M, Amon M. Influence of viscoelastic substances used in cataract surgery on corneal metabolism and endothelial morphology: comparison of Healon and Viscoat. J Cataract Refract Surg 2001;27:1756-61.

34. Brown JA. The role of hyaluronic acid in wound healing's proliferative phase. J Wound Care 2004;13:48-51.

35. Rivas Lacarte MP, Casasin T, Pumarola F, Alonso A. An alternative treatment for the reduction of tympanic membrane perforations: sodium hyaluronate. A double blind study. Acta Otolaryngol 1990;110:110-4.

36. Hellström S, Laurent C. Hyaluronan and healing of tympanic membrane perforations. An experimental study. Acta Otolaryngol Suppl 1987;442:54-61.

37. Ozturk K, Yaman H, Cihat Avunduk M, Arbag H, Keles B, Uyar Y. Effectiveness of MeroGel hyaluronic acid on tympanic membrane perforations. Acta Otolaryngol 2006;126:1158-63.

38. Kaur K, Singh H, Singh M. Repair of tympanic membrane perforation by topical application of $1 \%$ sodium hyaluronate. Indian J Otolaryngol Head Neck Surg 2006;58:241-4.

39. Rivas Lacarte MP, Casasin T, Alonso A. Effects of sodium hyaluronate on tympanic membrane perforations. J Int Med Res 1992;20:353-9.

40. Okan D, Woo K, Ayello EA, Sibbald G. The role of moisture balance in wound healing. Adv Skin Wound Care 2007;20:39-53; quiz 53-5. 